\title{
Editorial: Gravitational Waves: A New Window to the Universe
}

\author{
Rosalba Perna ${ }^{1}$ and Bruno Giacomazzo ${ }^{2,3 *}$ \\ ${ }^{1}$ Department of Physics and Astronomy, Stony Brook University, Stony Brook, NY, United States, ${ }^{2}$ Department of Physics \\ "Giuseppe Occhialini", University of Milano-Bicocca, Milan, Italy, ${ }^{3}$ INFN, Division of Milano-Bicocca, Milan, Italy
}

Keywords: gravitational waves, gamma-ray burst, kilonovae, numerical relativity, neutron star, black hole

\section{Editorial on the Research Topic}

\section{Gravitational Waves: A New Window to the Universe}

This Research Topic focuses on gravitational waves, and their role in advancing knowledge in research areas ranging from cosmology to high energy physics to nuclear physics.

The first article of this Research Topic (Palenzuela) provides a short introduction to the field of numerical relativity, including also a summary of the $3+1$ decomposition of Einstein's equations, allowing the reader to get a broad overview of the equations and numerical methods used in this field. Numerical relativity is one of the fundamental tools for the study of compact binary mergers and it has allowed for the study of gravitational wave sources, such as the ones discussed in the following articles.

Ciolfi and Foucart present an overview of the state of the art in the field of binaries composed of two neutron stars (NSs) or of an NS and a black hole (BH). Neutron star binaries have gained a lot of attention since the detection of the first gravitational wave (GW) signal emitted by one of such systems (GW170817). Observations of an electromagnetic counterpart have provided evidence for the presence of a relativistic jet and for the ejection of high-density NS material. The formation of a jet, and the properties of the ejecta have been the subject of numerous investigations, and they are reviewed in Ciolfi.

Similarly, in Foucart the reader will find an overview of NS-BH mergers and in particular of what theoretical models predict for their GW and electromagnetic (EM) emission. In the case of NS-BH mergers it is critical for the NS to be tidally disrupted by the companion $\mathrm{BH}$ in order to be able to produce bright EM signals and this is strongly correlated with the binary properties, including the NS equation of state.

One of the most detected sources of GWs is given by binary BH mergers and these are discussed in detail in Schmidt. This review describes both how these sources can be modeled from a theoretical point of view as well as the properties, such as masses and spins, of the many binary $\mathrm{BH}$ systems detected by the LIGO-Virgo Collaboration.

Among the GW sources detected by LIGO/Virgo to date, GW170817 was especially important since it was accompanied by electromagnetic radiation covering a very broad spectrum, from gamma-rays to radio. Detailed modeling of both the prompt (gamma-ray) emission and the following longer-wavelength radiations demonstrated that the properties of this source were consistent with those of a classical short gamma-ray burst (SGRB), hence firmly establishing the link between those and an NS-NS merger. Given the importance of this finding, Lazzati is specifically devoted to a review of the source GW170817 and its connection to SGRBs.

Another important source of EM emission from a binary NS merger is given by the so-called kilonova, described in Barnes. With respect to SGRBs, kilonovae have the advantage of being isotropic emitters and therefore may be detected with most binary NS mergers. They also constitute the site for the production of heavy elements. Barnes provides a review of the theoretical models 
used to predict the heavy element production and observational appearance, and what we have learned from their first observation associated with GW170817.

The last two articles are dedicated to an overview of the formation channels of solar mass binary $\mathrm{BH}$ systems (Mapelli) and of future detections of supermassive BHs with LISA (Sesana). More specifically, Mapelli reviews the two main channels of $\mathrm{BH}-\mathrm{BH}$ formation, that is the isolated one resulting from standard binary evolution, and the dynamical one, in which binaries are formed as a results of gravitational interactions in dense environments.

Looking into the future of gravitational wave science, Sesana provides a broad overview of the potential science with the Laser Interferometer Space Antenna (LISA). Expected to be launched in the 30s, GW detections with this instrument are expected to open an additional window into the Universe, and especially in the way the enigmatic supermassive BHs lurking in the center of galaxies were assembled.

\section{AUTHOR CONTRIBUTIONS}

RP and BG are the editors of this Research Topic.

\section{FUNDING}

RP acknowledged the support by NSF award \#AST-2006839.

Conflict of Interest: The authors declare that the research was conducted in the absence of any commercial or financial relationships that could be construed as a potential conflict of interest.

Copyright (๑) 2021 Perna and Giacomazzo. This is an open-access article distributed under the terms of the Creative Commons Attribution License (CC BY). The use, distribution or reproduction in other forums is permitted, provided the original author(s) and the copyright owner(s) are credited and that the original publication in this journal is cited, in accordance with accepted academic practice. No use, distribution or reproduction is permitted which does not comply with these terms. 